\title{
Analysis and Study of the Nonlinear Electrodynamics Behaviour of a Micro-beam Made of Ionic Polymer Metal Composite (IPMC)
}

\author{
Mohammad Reza Salehi Kolahi*, Hossein Moinekhah \\ Department of Mechanical and Chemistry Engineering, University of Sistan and Baluchestan, Zahedan, Iran \\ Email address: \\ Mohammad.salehi1370@eng.usb.ac.ir (M. R. S. Kolahi), hmoein aeng.usb.ac.ir (H. Moinekhah) \\ ${ }^{*}$ Corresponding author
}

\section{To cite this article:}

Mohammad Reza Salehi Kolahi, Hossein Moinekhah. Analysis and Study of the Nonlinear Electrodynamics Behaviour of a Micro-beam Made of Ionic Polymer Metal Composite (IPMC). Chemical and Biomolecular Engineering. Vol. 5, No. 1, 2020, pp. 15-20.

doi: $10.11648 /$ j.cbe. 20200501.13

Received: December 15, 2019; Accepted: January 7, 2020; Published: January 17, 2020

\begin{abstract}
Ionic Polymer Metal Composites (IPMCs) are a group of electroactive polymer materials that exhibit a large deformation due to the application of low voltages resulting from the movement of cations inside the polymer. These materials have many applications in various fields such as Micro-robotics, biomedical engineering equipment and artificial muscles. Due to the possibility of producing these materials in micro dimensions they can also be used in micro-electromechanical systems. On the other hand, due to their sensitivity to very low voltages, they can be a good substitute for silicon in micro-electromechanical systems. In the present study, the dynamical analysis of a micro-beam fixed at two ends made of these materials is investigated using electrical-chemical-mechanical relations. COMSOL Multiphysics software is used to solve the relations. The results showed that for harmonic stimulation (sinusoidal voltage) the system experiences only the same form of the first mode. It was also observed that increasing the frequency would decrease the amplitude of the micro-beam oscillation.
\end{abstract}

Keywords: Ionic Polymer Metal Composites, Micro-beam, Physical Model, COMSOL Multiphysics, Micro-electromechanical Systems

\section{Introduction}

There are many type of composites which have been used in different aspects [1], like Nanocomposites [2], Ionic polymer composites and so forth. Ionic Polymer Metal Composites (IPMCs) are a new group of polymer electroactive materials, which represent large deformations when exposed to a small electric field (zero to five volts). Recently, polymer electroactive materials have been extensively studied for their wide application in various industries such as robotics, biomedical engineering and artificial muscles [3-6]. This material was first discovered by Shahinpoor in 1992 and then formally introduced in 1998 [7]. IPMCs are consisted of a polymer membrane surrounded by two metal electrodes, that can be produced in various sizes and dimensions depending on the intended use. Polymer membranes are usually made of Nafion or Flemion and the electrodes are made of high conductivity metals such as platinum and gold. IPMCs are intelligent materials and their deformation results from the application of voltage to two electrodes, which leads to an electric field inside the polymer membrane causing the movement of ions [8-9]. These materials are also used as sensors, which by forcing mechanical deformation into the fragment, the ions are imposed to move, thereby generating the potential difference between the two electrodes [10].

Although the above materials have found increased use in various industries and their scope of application is rising dramatically, researchers are still investigating the identification and prediction of their behavior against different input voltages with different frequencies. Hence, different models have been developed to identify the behavior and performance of these materials, which can be divided into three general categories: 1- Black Box Model, 2- Gray Box 
Model and 3- White Box Model (Physical Model). In the black box model, the model is based solely on the relations between inputs and system response. In this case, the model is strongly dependent on the sample being studied and the test conditions. Therefore, the more experiments in this model, the more reliable results will be. And in the gray box model, the material parameters are obtained based on the experimental results. These parameters represent physical concepts with a process. But in the physical model, which is the most developed and sophisticated method yet for detecting the behavior of an IPMC actuator, the laws governing the transfer of ions as well as the electrical-mechanical and electrical-chemical effects are examined. To model these materials in this way, differential equations with partial derivatives must be analyzed [11].

Annabestani et al. (2013) used a black box model to identify the behavior of actuator made of IPMCs [12]. Their model also included 2 main sections. One part consisted of a fuzzy-neural adaptive system and the other consisted of a retrograde automatic system with nonlinear external input. Caponetto et al (2014) proposed a gray box model to identify the behavior of the target actuators [13]. They modeled the nonlinear properties of this type of operator with an electric circuit. They also used single and multi-objective optimization functions to estimate the parameters of their model. They used the Genetic Algorithm (GA) to compare the displacement of their proposed model and of the actual operator and tried to minimize the error with GA. Micro-beams are used as resonators, optical switches, micro-pumps and so on in micro-electromechanical systems. In most of these systems, the micro-beam is made of silicon, which requires high voltages to stimulate. When the voltage reaches a certain value, the micro-beam's internal forces are out of equilibrium with the Coulomb pressure, causing the micro-beam to be drawn toward the fixed electrode (called Pull-in phenomenon) and be in contact with it and become disrupted [14-15]. Due to the excitation mechanism of the IPMC, it can be concluded that the use of these materials in micro-electromechanical systems can eliminate the Pull-in instability.

Black and gray box models are valid only for the sample tested and are not scalable. For example, if the sample length changes in black or gray box model, it cannot predict the operator's behavior against different voltages [16]. According to this point, a physical model based on electrochemical equations has been used in this study to analyze and predict the dynamic behavior of IPMC actuator The governing equations are solved using the Finite Element Method (FEM) by COMSOL Multiphysics software. Studies show that this method has not been used so far for an IPMC micro-beam analysis. Many research have been conducted in this field as Esmaeili et al in 2016 has worked on Wave function properties of a single and a system of magnetic flux tube [17]. There are different methods to solve this issue, Mohammadzadeh et al. suggest particle swarm optimization method [18-19] or Afshar implements Population Balance Modeling (PBM) and
Initial Development Method (IDM) [20-21] on the other hand Azarang et al. describe about Laplacian-based and Nonfragile fuzzy method [22-24]. Ramtin et al. have studied Self-stabilizing algorithms [25-27]. In which different materials have been derived, such as coatings by non-isocyanate polyurethanes [28-31].

Khiabani et al. have conducted research on design and implementation of an optimal switching controller based on State-Dependent Riccati equation [32-34]. Razzaghi has worked on nonlinear dynamics and control of an inertially actuated jumper robot [35-36], and on the following Hayati et al. have studied different methods like Low-power and CMOS burst-mode [37-38], dynamic addressing by Ghayouraneh et al. who have worked on Holistic optimization technique for solving low thermal conductivity [39-43]. Many other researches have been conducted in this field by Gharib [44] in quantitative-fuzzy Controller Design for Multivariable Systems with Uncertainty, and Kondori [45] in identifying catalytic active sites of trimolybdenum phosphide (Mo3p) for electrochemical hydrogen evolution and Ranjbar [46-48] in laser-induced studies. Hemmati et al. [49-51] have used metaheuristic optimization techniques for solving problems in this field. Evaluating the stability of specific adhesion of particles to membranes in simple shear flow is what have been studied by Sarvestani et al. [52-54]. The damage of beams is studied by Moghaddam et al. [55-56] by signal processing based approach for damage. Evaluating the risk of accident and damages has been studied by Movahedi et al. [57-58]. One of the main aspects of this study is nonlinear behavior and optimization, many ways are considered to be done like digital comparative holography which have been studied by Ahmadzadegan et al. [59-62].

\section{Modeling and Governing Equations}

The figure 1 shows a schematic diagram of an IPMC micro-beam (fixed at both ends). We assume that the micro-beam is homogeneous and isotropic with linear elasticity, L length, A the area of rectangular cross section, b width and thickness of $d$.

According to Figure 1, by applying a voltage to two electrodes and generating an electric field within the polymer membrane, free cations begin to move toward the cathode, which causes swelling in the cathode and shrinkage at the anode side, resulting in a bending motion in the actuator. It should be noted that anions are stable and constitute the polymer membrane. The transfer of cations is described by the Nernst-Planck equation [63].

$$
\frac{\partial C}{\partial t}+\nabla \cdot(-D \nabla C-z \mu F C \nabla \varphi)=0
$$

In the above relation $\mathrm{C}$ is the cation concentration, $\mu$ cations transferability, D diffusivity constant, F Faraday constant, z charge number and $\varphi$ electric potential within the polymer layer. The electrical potential inside the polymer membrane $(\varphi)$ differs from the electrical potential inside the electrode $(\mathrm{V}) . \varphi$ 
is described using Poisson's equation [63].

$$
\nabla . \breve{E}=-\nabla^{2} \varphi=\frac{\rho}{\varepsilon}
$$

Where $\varepsilon$ is the permeability coefficient and $\rho$ is the charge density inside the polymer membrane and is defined as follows [63].

$$
\rho=F\left(C-C_{0}\right)
$$

Where $\mathrm{C}_{0}$ is the initial concentration of the cation, and the Ohm's Law is used to define the electrical potential inside the electrodes (V) [63].

$$
\sigma \nabla V=-\breve{\jmath}
$$

Where $\sigma$ is the electrical conductivity of the electrodes and $\mathrm{j}$ the current density inside the electrodes. Now one can establish a relation between the electrical potential at the electrodes and the electrical potential at the polymer membrane. Such a relation can be obtained by using Gauss's law [64].

$$
j_{I}=-b \varepsilon \frac{\partial \varphi}{\partial t} \cdot n^{r}
$$

In the above relation $\mathrm{n}$ is the normal vector of the interface between the polymer membrane and the electrodes. By coupling and simultaneously solving the above equations in COMSOL, the charge density inside the polymer membrane $\rho$ is obtained. Nemat-Nasser (2) showed that the deformation of an IPMC can be attributed to a charge-proportional force.

$$
f_{z}=\alpha \rho
$$

The $\alpha$ coefficient can be calculated by experiment. Now with the force $f$ and using the elasticity equation one can calculate the displacement field.

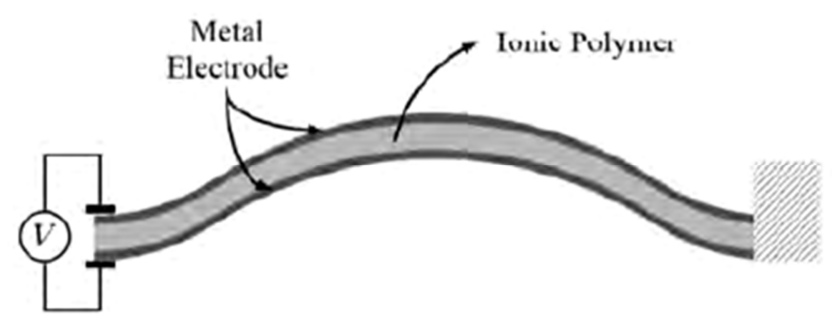

Figure 1. Schematic model of a micro-beam of IPMC in a micro-electromechanical system.

\section{Results}

To ensure the accuracy of FEM modeling, the tip displacement of an IPMC strip of $51,07 \times 9,94 \times 0.582 \mathrm{~mm}^{3}$ is considered to be applied by a sinusoidal voltage and acting at $4 \mathrm{~V}$ and $1 \mathrm{~Hz}$. The simulation constants are given in Table 1. As can be seen in Figure 2, a good agreement is observed between the present modeling and the research of Mr. Shen et al. [65].

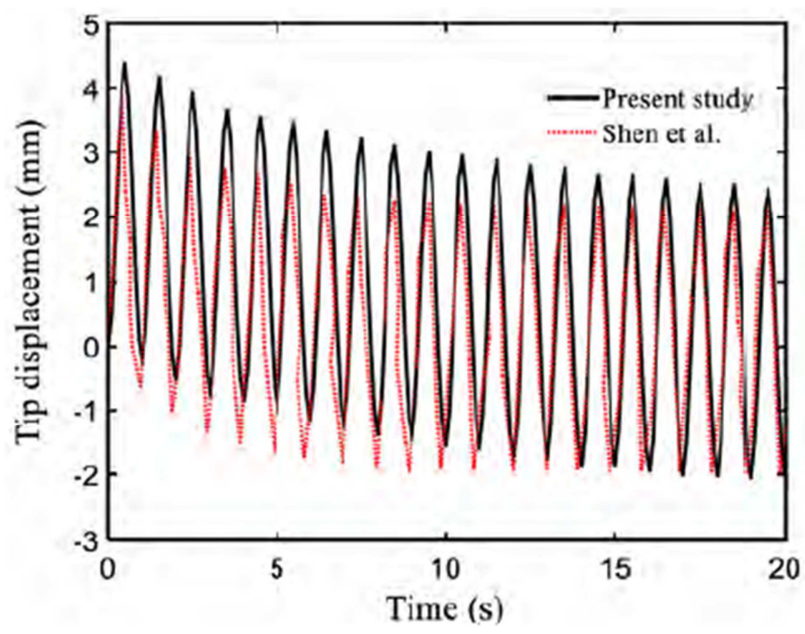

Figure 2. Time history graph of a single-end fixed IPMC strip.

Table 1. Constants used for the simulation.

\begin{tabular}{llll}
\hline Row & Quantity & Unit & Value \\
\hline 1 & D diffusivity constant & $\mathrm{m} / \mathrm{s}^{2}$ & $7 \times 10^{12}$ \\
2 & C $_{0}$ initial concentration & $\mathrm{mol} / \mathrm{m}^{3}$ & 1200 \\
3 & F Faraday constant & $\mathrm{C} / \mathrm{mol}^{2}$ & 96485 \\
4 & $\mu$ cations transferability & $\mathrm{s} . \mathrm{mol} / \mathrm{kg}$ & $2.9 \times 10^{-15}$ \\
5 & Z Charge value & - & 1 \\
6 & $\varepsilon$ permeability coefficient & $\mathrm{F} / \mathrm{m}$ & 0.0002 \\
7 & $\sigma$ electrical conductivity & $\mathrm{S} / \mathrm{m}$ & $4.65 \times 10^{12}$ \\
8 & E Young Modulus & $\mathrm{MPa}$ & 90.92 \\
9 & $\rho_{m}$ Composite density & $\mathrm{kg} / \mathrm{m}^{3}$ & 784 \\
10 & v Poisson's ratio & - & 0.49 \\
11 & $\alpha$ Proportionality constant & $\mathrm{J} / \mathrm{C}$ & $7.5 \times 10^{-8}$ \\
\hline
\end{tabular}

It should be noted that by using micromachining technologies it is possible to fabricate micro-beam out of IPMC [65]. Figure 3 shows the time history diagram for a fixed two-end micro-beam of $1000 \times 50 \times 30 \mu \mathrm{m}^{3} 3$ dimensions [65]. The applied voltage is sinusoidal with an amplitude of 2 $\mathrm{V}$ and a frequency of $1 \mathrm{~Hz}$.

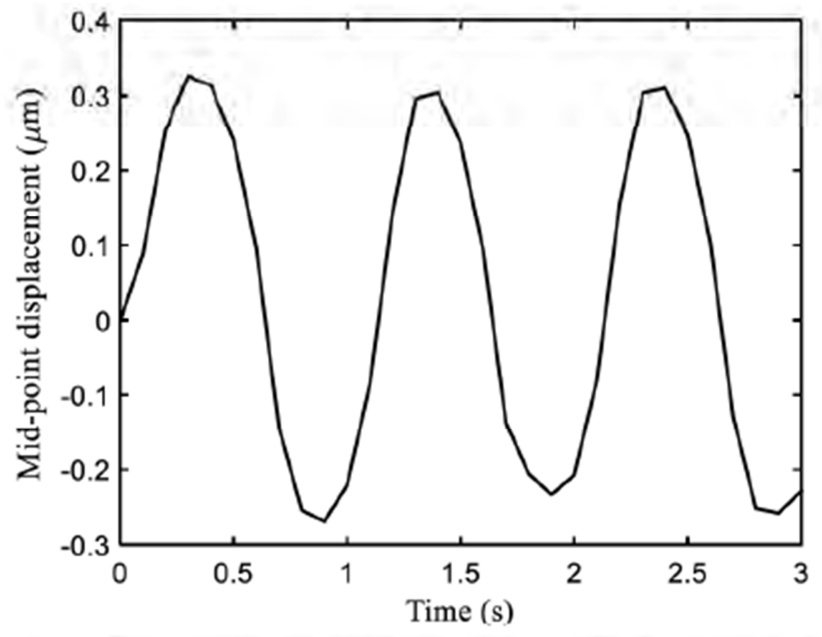

Figure 3. Time history diagram of a fixed two-end IPMC micro-beam.

Figure 4 shows the micro-beam deformation for a cycle of oscillation, and as can be seen the system experiences only the first mode shape.

Figure 5 shows the micro-beam frequency response for a 
sinusoidal voltage of 1 and $2 \mathrm{~V}$ amplitudes.

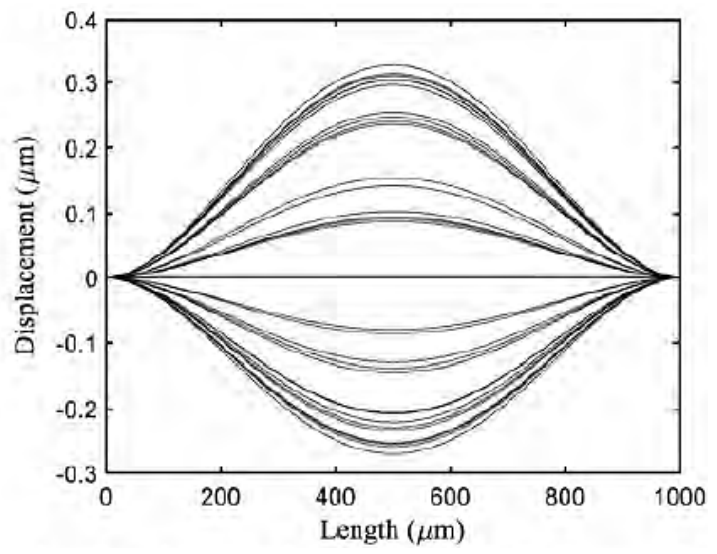

Figure 4. Micro-beam deformation for a cycle of oscillation, sinusoidal voltage with amplitude of $2 \mathrm{~V}$ and a frequency of $1 \mathrm{~Hz}$.

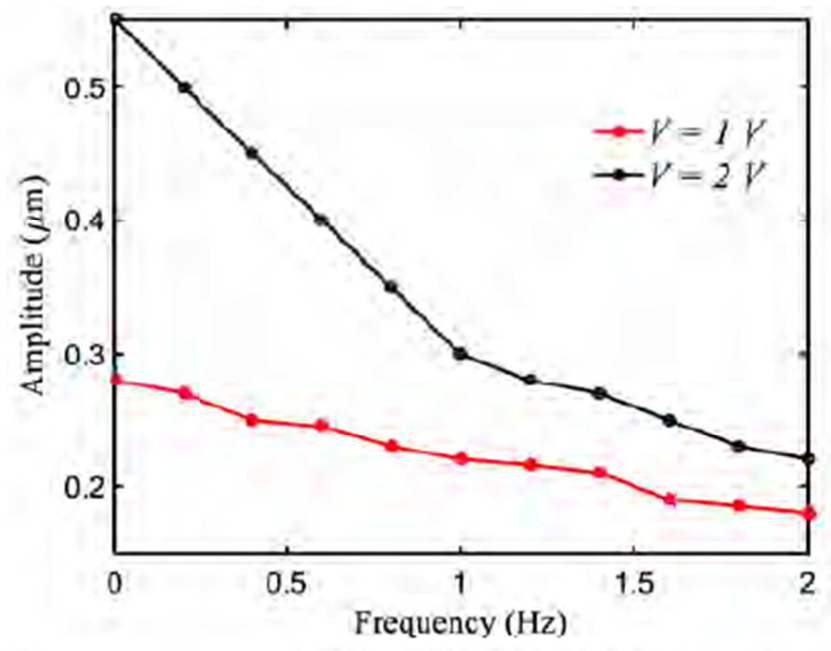

Figure 5. Frequency response of the micro-beam.

As it can be seen in Figure 5, for a $1 \mathrm{~V}$ amplitude, the frequency change does not have much effect on the oscillation domain. On the other hand, it is observed that with increasing frequency the oscillation amplitude decreases.

\section{Conclusion}

In this study, the electro-dynamic behavior of a micro-beam of these materials is investigated using electrical chemical mechanical relationships governing the behavior of IPMCs. Governing equations are solved using four software modules (1-electric currents, 2-particle transport, 3-Partial differential equations, 4-structural mechanics), and the results show that for the harmonic excitation (sinusoidal voltage) the system only experiences the first mode shape, it was also observed that increasing the excitation frequency leads to a decrease in excitation amplitude. Comparing the results of the present study with some research in the field of silicon micro-beams $(10,11)$ leads to a conclusion that IPMCs can replace silicon in micro-electromechanical systems. With the advantage that the voltage and frequency needed to excite them are much lower than for silicon. On the other hand, this replacement can lead to the removal of pull-in instability from micro-electromechanical systems.

\section{References}

[1] Ghadimi, A., \& Asadi, S. (2018). Modelling of composite right/left-handed active multiconductor transmission lines (AMCTL) in time domain. International Journal of Numerical Modelling: Electronic Networks, Devices and Fields, 31 (1), e2257

[2] Chegeni, M., Pour, S. K., \& Dizaji, B. F. (2019). Synthesis and characterization of novel antibacterial Sol-gel derived $\mathrm{TiO}_{2} / \mathrm{Zn}_{2}$ $\mathrm{TiO}_{4} / \mathrm{Ag}$ nanocomposite as an active agent in Sunscreens. Ceramics International, 45 (18), 24413-24418. doi: 10.1016/j.ceramint.2019.08.163

[3] Shahinpoor, M., \& Kim, K. J. (2001). Ionic polymer-metal composites: I. Fundamentals. Smart materials and structures, $10(4), 819$.

[4] Nemat-Nasser, S., \& Li, J. Y. (2000). Electromechanical response of ionic polymer-metal composites. Journal of Applied Physics, 87 (7), 3321-3331.

[5] Del Bakhshayesh, A. R., Asadi, N., Alihemmati, A., Nasrabadi, H. T., Montaseri, A., Davaran, S.,... \& Abedelahi, A. (2019). An overview of advanced biocompatible and biomimetic materials for creation of replacement structures in the musculoskeletal systems: focusing on cartilage tissue engineering. Journal of biological engineering, 13 (1), 85.

[6] Hosseini, V., Maroufi, N. F., Saghati, S., Asadi, N., Darabi, M., Ahmad, S. N. S.,... \& Rahbarghazi, R. (2019). Current progress in hepatic tissue regeneration by tissue engineering. Journal of translational medicine, 17 (1), 383.

[7] Shahinpoor, M. and Kim, K. J.. 2001. "Ionic polymer-metal composites: I. Fundamentals". Smart materials and structures, 10 (4), Aug, pp. 819.

[8] Kim, K. J. and Shahinpoor. M., 2002. "A novel method of tnanufixturing three-dimensional ionic polymer-metal composites (IPMCs) biomitnetic sensors. actuators and artificial muscles". Polymer, 43 (3), Feb, pp. 797-802.

[9] Kim, K. J. and Shahinpoor, M., 2003. "Ionic polymer-metal composites: II. Manufacturing techniques". Smart materials and structures, 12 (1), Jan, pp. 65.

[10] Punning, A., Kruusmaa, M. and Aabloo, A., 2007. "A self-sensing ion conducting polymer metal composite (IPMC) actuator". Sensors and Achialors A: Physical, 136 (2), May. pp. 656-664.

[11] Moeinkhah, H., Reneepazhand, J, and Akbarzadeh, A., 2013. "Analytical dynamic modeling of a cantilever IPMC actuator based on a distributed electrical circuit". Smart Materials and Structures, 22 (5), Apr, pp. 055033.

[12] Annabestani, M. and Naghavi, N., 2014. "Nonlinear identification of PMC actuators based on ANFIS - NARX paradigm". Sensors and Actuators A: Physical, 209 (1), Mar, pp. 140-148.

[13] Caponetto, R., Graziani, S., Pappalardo, F. and Sapuppo, F., 2014. "Identification of IPMC nonlinear model via single and multi-objective optimization algorithms". ISA transactions, 53 (2), Mar, pp. 481-488. 
[14] Hu, Y. J., Yang, J. and Kitipornchai, S., 2013. "Snap-through and pull-in analysis of an clectro-dynamically actuated curved micro-beam using a nonlinear beam model". Journal of Sound and Vibration, 332 (15), Jul, pp. 3821-3832.

[15] Hsu, T. R., 2008. MEMS and Microsystems: Design, iidangfacture, and Nanoscale Engineering, John Wiley \& Sons, New Jersey.

[16] Chen, Z., 2009. "Ionic polymer-metal composite artificial muscles and sensors: A control systems perspective". PhD Thesis, Michigan State University. Michigan.

[17] Esmaeili, S., Nasiri, M., Dadashi, N., \& Safari, H. (2016). Wave function properties of a single and a system of magnetic flux tube (s) oscillations. Journal of Geophysical Research: Space Physics, 121 (10), 9340-9355.

[18] Mohammadzadeh, A., Ghoddoosian, A., \& Noori-Damghani, M. (2011). Balancing of the flexible rotors with particle swarm optimization method. International Review of Mechanical Engineering, 5 (3), 490-496.

[19] Damghani, Mohammad Nouri, and Arash Mohammadzadeh Gonabadi. "Numerical Study of Energy Absorption in Aluminum Foam Sandwich Panel Structures Using Drop Hammer Test." Journal of Sandwich Structures \& Materials, vol. 21, no. 1, Jan. 2019, pp. 3-18, doi: $10.1177 / 1099636216685315$.

[20] Afshar, A., Hosseini, M. S., \& Behzadfar, E. (2014). Numerical study of the agglomerates dispersion behavior in shear and elongational flow fields in viscous media using Population Balance Modeling (PBM). Scientia Iranica. Transaction C, Chemistry, Chemical Engineering, 21 (6), 2107.

[21] Thompson, D. S., Meng, D., Afshar, A., Bassou, R., Zong, J., Bonaccurso, E.,... \& Vercillo, V. (2018). Initial Development of a Model to Predict Impact Ice Adhesion Stress. In 2018 Atmospheric and Space Environments Conference (p. 3344).

[22] Azarang, A., Kamaei, S., Miri, M., \& Asemani, M. H. (2016). A new fractional-order chaotic system and its synchronization via Lyapunov and improved Laplacian-based method. Optik, 127 (24), 11717-11731.

[23] Azarang, A., Ranjbar, J., Mohseni, H., \& Andy, M. A. (2017). Output feedback synchronization of a novel chaotic system and its application in secure communication. International Journal of Computer Science and Network Security, 17, 72-77.

[24] Azarang, A., Miri, M., Kamaei, S., \& Asemani, M. H. (2018). Nonfragile fuzzy output feedback synchronization of a new chaotic system: design and implementation. Journal of Computational and Nonlinear Dynamics, 13 (1), 011008.

[25] Ramtin, A., Hakami, V., \& Dehghan, M. (2013, December). A Perturbation-Proof Self-stabilizing Algorithm for Constructing Virtual Backbones in Wireless Ad-Hoc Networks. In International Symposium on Computer. Networks and Distributed Systems (pp. 66-76). Springer, Cham

[26] Ramtin, A., Hakami, V., \& Dehghan, M. (2014, September). A self-stabilizing clustering algorithm with fault-containment feature for wireless sensor networks. In 7'th International Symposium on Telecommunications (IST'2014) (pp. 735-739). IEEE.

[27] Ramtin, A., Hakami, V., \& Dehghan, M. (2014, May). Self-stabilizing algorithms of constructing virtual backbone in selfish wireless ad-hoc networks. In 2014 22nd Iranian Conference on Electrical Engineering (ICEE) (pp. 914- 919). IEEE.
[28] Zareanshahraki, F., \& Mannari, V. (2018). "Green" UV-LED gel nail polishes from bio-based materials. International journal of cosmetic science, 40 (6), 555-564.

[29] Karami, Z., Kabiri, K., \& Zohuriaan-Mehr, M. J. (2019). Non-isocyanate polyurethane thermoset based on a bio-resourced star-shaped epoxy macromonomer in comparison with a cyclocarbonate fossil-based epoxy resin: A preliminary study on thermo-mechanical and antibacterial properties. Journal of $\mathrm{CO}_{2}$ Utilization, 34, 558-567.

[30] Ghasemlou, M., Daver, F., Ivanova, E. P., \& Adhikari, B. (2020). Synthesis of green hybrid materials using starch and non-isocyanate polyurethanes. Carbohydrate Polymers, 229, 115535 .

[31] Akdogan, O. K., Zareanshahraki, F., \& Mannari, V. (2019). Dual-cure polyurethane coatings from soybean oil and their film properties as a function of cure sequence. Eur. J. Lipid Sci. Technol., 50 (4), 112-122.

[32] Khiabani, A. G., \& Heydari, A. (2019). Design and implementation of an optimal switching controller for uninterruptible powersupply inverters using adaptive dynamic programming. IET Power Electronics

[33] Khiabani, A. G., \& Heydari, A. (2018, September). Optimal switching of voltage source inverters using approximate dynamic programming. In ASME 2018 Dynamic Systems and Control Conference (pp. V001T01A006-V001T01A006). American Society of Mechanical Engineers.

[34] Babazadeh, R., \& Khiabani, A. G. (2018, October). Nonlinear Observer Design for RC Battery Model for Estimating State of Charge \& State of Health Based on State-Dependent Riccati Equation. In 2018 IEEE Electrical Power and Energy Conference (EPEC) (pp. 1-5). IEEE.

[35] Razzaghi, P., Al Khatib, E., \& Bakhtiari, S. (2019). Sliding mode and SDRE control laws on a tethered satellite system to de-orbit space debris. Advances in Space Research, 64 (1), $18-27$

[36] Razzaghi, P., Al Khatib, E., \& Hurmuzlu, Y. (2019). Nonlinear dynamics and control of an inertially actuated jumper robot. Nonlinear Dynamics, 1-16.

[37] Hayati, H., \& Ehsanian, M. (2014, December). Low-power burst-mode clock recovery circuit using analog phase interpolator. In 2014 26th International Conference on Microelectronics (ICM) (pp. 120-123). IEEE.

[38] Hayati, H., \& Ehsanian, M. (2015). A 5-Gbps CMOS Burst-Mode CDR Circuit With an Analog Phase Interpolator for PONs. Informacije MIDEM, 45 (1), 39-46.

[39] Avval, A. G., \& El-Ghazaly, S. M. (2018, April). Holistic optimization technique for solving low thermal conductivity of sapphire substrates in high frequency devices. In 2018 Texas Symposium on Wireless and Microwave Circuits and Systems (WMCS) (pp. 1-4). IEEE.

[40] A. G. Avval, E. Larique, S. M. El-Ghazaly, "Heterojunction field effect transistors" in Reference Module in Materials Science and Materials Engineering, Elsevier, 2018.

[41] Ghayouraneh, S., El-Ghazaly, S. M., \& Rankin, J. M. (2018). Dynamic Addressing for On-Demand Mobility. In 2018 Aviation Technology, Integration, and Operations Conference (p. 4152). 
[42] Ghayouraneh, S., Rankin, J. M., \& El-Ghazaly, S. M. (2019). Practical Considerations in Traffic Flow Systems for ODM Vehicles. In 2019 Integrated Communications, Navigation and Surveillance Conference (ICNS) (pp. 1-7). IEEE.

[43] Ghayouraneh, S., El-Ghazaly, S., \& Rankin, J. (2019). Investigation of Traffic Flow for ODM Vehicles in Dynamic ICAO Addressing Process. In AIAA Aviation 2019 Forum (p. 3627).

[44] Gharib, M. R., \& Daneshvar, A. (2019). Quantitative-fuzzy Controller Design for Multivariable Systems with Uncertainty. International Journal of Control, Automation and Systems, 17 (6), 1515-1523.

[45] Kondori, A., Esmaeilirad, M., Baskin, A., Song, B., Wei, J., Chen, W.,... \& Asadi, M. (2019). Identifying Catalytic Active Sites of Trimolybdenum Phosphide (Mo3P) for Electrochemical Hydrogen Evolution. Advanced Energy Materials, 9 (22), 1900516.

[46] Ranjbar O. A., A. N. Volkov, Kinetic and hydrodynamic simulations of laser-induced plume expansion, Early Career Tech. J. 15 (2016) 160-167

[47] Ranjbar, O. A., Lin, Z., \& Volkov, A. N. (2018). One dimensional kinetic simulations of plume expansion induced by multi-pulse laser irradiation in the burst mode at $266 \mathrm{~nm}$ wavelength. Vacuum, 157, 361-375

[48] Palya, A., Ranjbar, O. A., Lin, Z., \& Volkov, A. N. (2018). Effect of the background gas pressure on the effectiveness of laser-induced material removal from deep cavities in irradiated targets. Applied Physics A, 124 (1), 32.

[49] Hemmati, R., \& Rahideh, A. (2017). Optimal design of slotless tubular linear brushless PM machines using metaheuristic optimization techniques. Journal of Intelligent \& Fuzzy Systems, 32 (1), 351-362.

[50] Hemmati, R., Wu, F., \& El-Refaie, A. (2019, May). Survey of Insulation Systems in Electrical Machines. In 2019 IEEE International Electric Machines \& Drives Conference (IEMDC) (pp. 2069-2076). IEEE.

[51] Nikoobakht, A., Aghaei, J., Fallahzadeh-Abarghouei, H., \& Hemmati, R. (2019). Flexible Co-Scheduling of Integrated Electrical and Gas Energy Networks under Continuous and Discrete Uncertainties. Energy.

[52] Tehrani, M., Moshaei, M. H., \& Sarvestani, A. S. (2017). Network polydispersity and deformation-induced damage in filled elastomers. Macromolecular Theory and Simulations, 26 (6), 1700038

[53] Moshaei, M. H., Tehrani, M., \& Sarvestani, A. (2019). On stability of specific adhesion of particles to membranes in simple shear flow. Journal of biomechanical engineering, 141 (1), 011005.
[54] Moshaei, M. H., Tehrani, M., \& Sarvestani, A. (2019). Rolling adhesion of leukocytes on soft substrates: Does substrate stiffness matter?. Journal of biomechanics

[55] Moghaddam, H., Hajirasouliha, I., \& Doostan, A. (2005). Optimum seismic design of concentrically braced steel frames: concepts and design procedures. Journal of Constructional Steel Research, 61 (2), 151-166.

[56] Moghaddam, H., \& Hajirasouliha, I. (2009). On the optimum performance-based design of structures. In Proceedings of a US-Iran Seismic Workshop, Irvine, California, PEER report (Vol. 2).

[57] Movahedi, N., Linul, E., \& Marsavina, L. (2018). The temperature effect on the compressive behavior of closed-cell aluminum-alloy foams. Journal of Materials Engineering and Performance, 27 (1), 99-108.

[58] Movahedi, N., Taherishargh, M., Belova, I. V., Murch, G. E., \& Fiedler, T. (2019). Mechanical and Microstructural Characterization of an AZ91-Activated Carbon Syntactic Foam. Materials, 12 (1), 3.

[59] Ahmadzadegan, M. H., KINNUNEN, M., \& Fabritius, T. (2014). Ant colony optimization approach to digital comparative holography through traveling salesman problem. Optoelectronics and Advanced Materials-Rapid Communications, 8 (November-December 2014), 1246- 1249.

[60] Ahmadzadegan, M. H., \& Fabritius, T. (2013, August). Kiyotaki-Moore approach to wavefront manipulation in digital comparative holography. In 2013 3rd International Conference on Instrumentation Control and Automation (ICA) (pp. 203-206). IEEE

[61] Ahmadzadegan, M. H., Fabritius, T., \& Notare, M. S. M. A. (2014). Chaotic analysis of optical vignetting in digital comparative holography. IEEE Latin America Transactions, 12 (2), 244-247.

[62] Ahmadzadegan, M. H., \& Fabritius, T. (2014, October). Percolation approach to digital comparative holography via optical flow investigation. In 2014 4th International Conference on Computer and Knowledge Engineering (ICCKE) (pp. 200-203). IEEE.

[63] Pugal, D., Kim, K. J. and Aabloo, A., 2011. "An explicit physics-based model of ionic polymer-metal composite actuators". Journal ofApplied Physics. Oct, 110 (8), pp. 084904

[64] Pugal, D., 2012. Physics based model of ionic polymer-metal composite electromechanical and mechanoelectrical transduction. PhD Thesis, University of Nevada, Reno.

[65] Shen, Q., Palmre, V., Stalbaum, T., \& Kim, K. J. (2015). A comprehensive physics-based model encompassing variable surface resistance and underlying physics of ionic polymer-metal composite actuators. Journal of Applied Physics, 118 (12), 124904. 